\title{
lon-beam mixing in crystalline and amorphous germanium isotope multilayers
}

\author{
H. Bracht, ${ }^{1, a)}$ M. Radek, ${ }^{1}$ R. Kube, ${ }^{1}$ S. Knebel, ${ }^{1}$ M. Posselt, ${ }^{2}$ B. Schmidt, ${ }^{2}$ E. E. Haller, ${ }^{3}$ \\ and D. Bougeard ${ }^{4}$ \\ ${ }^{1}$ Institut für Materialphysik, Westfälische Wilhelms-Universität Münster, 48149 Münster, Germany \\ ${ }^{2}$ Helmholtz-Zentrum Dresden-Rossendorf, 01314 Dresden, Germany \\ ${ }^{3}$ Lawrence Berkeley National Laboratory, Berkeley, California 94720, USA \\ ${ }^{4}$ Institut für Experimentelle und Angewandte Physik, 93040 Regensburg, Germany
}

(Received 8 August 2011; accepted 29 September 2011; published online 1 November 2011)

Gallium (Ga) implantation induced self-atom mixing in crystalline and amorphous germanium $(\mathrm{Ge})$ is investigated utilizing isotopically controlled $\mathrm{Ge}$ multilayer structures grown by molecular beam epitaxy. The distribution of the Ga ions and the ion-beam induced depth-dependent mixing of the isotope structure was determined by means of secondary ion mass spectrometry. Whereas the distribution of $\mathrm{Ga}$ in the crystalline and amorphous $\mathrm{Ge}$ is very similar and accurately reproduced by computer simulations based on binary collision approximation (BCA), the ion-beam induced self-atom mixing is found to depend strongly on the state of the Ge structure. The experiments reveal stronger self-atom mixing in crystalline than in amorphous Ge. Atomistic simulations based on BCA reproduce the experimental results only when unphysically low $\mathrm{Ge}$ displacement energies are assumed. Analysis of the self-atom mixing induced by silicon implantation confirms the low displacement energy deduced within the BCA approach. This demonstrates that thermal spike mixing contributes significantly to the overall mixing of the Ge isotope structures. The disparity observed in the ion-beam mixing efficiency of crystalline and amorphous Ge indicates different dominant mixing mechanisms. We propose that self-atom mixing in crystalline $\mathrm{Ge}$ is mainly controlled by radiation enhanced diffusion during the early stage of mixing before the crystalline structure turns amorphous, whereas in an already amorphous state self-atom mixing is mediated by cooperative diffusion events. (C) 2011 American Institute of Physics. [doi:10.1063/1.3658259]

\section{INTRODUCTION}

Atomic mixing in solids caused by ion irradiation is a well-known phenomenon that has been considered in the framework of radiation effects in materials already for many years. $^{1-5}$ The understanding of ion-beam induced atomic mixing is crucial not only for ion-beam processing of semiconductor devices and other functional materials, but also for the evaluation of the depth resolution achieved with state of the art sputtering techniques. Modern electronic device fabrication involves ion implantation processes. With the continuing decrease of the lateral and vertical dimensions of electronic devices, atomic displacement induced by particle irradiation is important as this process alters, e.g., the distribution of dopants and the quality of $\mathrm{Si} / \mathrm{SiGe}$ heterointerfaces. ${ }^{6}$ Today dopant profiles and heterointerfaces are mainly analyzed with secondary ion mass spectrometry (SIMS). Since this technique also induces atomic mixing it overestimates, e.g., the intermixing at a $\mathrm{Si} / \mathrm{SiGe}$ heterointerface. In order to reduce the impact of atomic mixing in ion-beam processing and ion-beam analysis of semiconductor devices low primary ion energies in the range of a few $\mathrm{keV}$ and below $1 \mathrm{keV}$ are used. However, the threshold energies for the production of Frenkel pairs in $\mathrm{Si}(13 \mathrm{eV})$ and $\mathrm{Ge}(15 \mathrm{eV})$ are still well below the energy of the incoming ions (see,

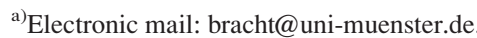

e.g., Ref. 5). Accordingly, even these low-energy ion beams cause atomic displacements and, finally, atomic mixing and defect formation.

Over the past decades data on ion-beam mixing in materials have become available mainly for metals and silicon. ${ }^{5}$ Data on atomic mixing mostly refer to ion-beam induced broadening of impurity profiles rather than to self-atom mixing. Although the latter experiment would be more fundamental as the intermixing of matrix atoms could be followed directly without any chemical effects involved, such studies would require labeling of host atoms using, e.g., radioactive or enriched stable isotopes. Due to the lack of appropriate samples such studies could not be performed extensively and only experiments on ion-beam mixing of isotopic copper ${ }^{7}$ and silver bi-layers ${ }^{8}$ were reported until recently. With the development in epitaxial deposition techniques and the availability of enriched stable isotopes of $\mathrm{Si}$ and $\mathrm{Ge}$, the first studies on ion-beam induced self-atom mixing in $\mathrm{Si}$ and $\mathrm{Ge}$ were recently reported. ${ }^{9,10}$

In this work we report experiments on gallium $(\mathrm{Ga})$ ion implantation induced mixing of crystalline and amorphous Ge isotope multilayer structures and on silicon ( $\mathrm{Si}$ ) implantation induced self-atom mixing in crystalline Ge. Whereas the distribution of $\mathrm{Ga}$ atoms after implantation in the crystalline and amorphous structure is very similar, the self-atom mixing strongly differs. The larger mixing of the crystalline compared to the amorphous isotope multilayer, which is at 
variance with the predictions of atomistic simulations in the binary collision approximation (BCA), not only indicates a contribution of thermal spike mixing but also suggests different mechanisms of mixing in the crystalline and amorphous Ge. Previous molecular dynamic (MD) simulations of Nordlund et al. ${ }^{11}$ also suggest a difference in mixing between amorphous and crystalline Ge but the mixing in amorphous Ge was found to be larger than in its crystalline counterpart.

\section{EXPERIMENTAL}

Crystalline $\left({ }^{\text {nat }} \mathrm{Ge} /{ }^{70} \mathrm{Ge}\right)_{10}$ and amorphous $\left({ }^{\text {nat }} \mathrm{Ge} /{ }^{73} \mathrm{Ge}\right)_{10}$ isotope multilayers were grown by means of molecular beam epitaxy (MBE). The thickness of each natural and isotopically enriched Ge layers is about $15 \mathrm{~nm}$. The crystalline structure was deposited on a (100)-oriented n-type Ge wafer with a specific resistivity of $40 \Omega \mathrm{cm}$. The amorphous structure was grown on top of a Ge wafer that prior to the MBE deposition was pre-amorphized to a depth of about $100 \mathrm{~nm}$ by implantation with $150 \mathrm{keV}$ Ge ions at a dose of $10^{15} \mathrm{~cm}^{-2}$. Figure 1 illustrates ${ }^{74} \mathrm{Ge}$ and ${ }^{70} \mathrm{Ge}$ concentration profiles of the crystalline and amorphous as-grown isotope structures, respectively, measured with SIMS. The depths of the craters left from the SIMS analyses were determined with a Talystep profilometer with an accuracy of about $5 \%$. The limited accuracy of the crater depth measurements is reflected in slightly different overall thicknesses of the in fact same isotope structure. The concentration depth profile of the as-grown multilayers is analytically described with the equation

$$
C_{\mathrm{Ge}}(x)=C_{1}+\frac{C_{2}-C_{1}}{2} \sum_{i=1}^{20}(-1)^{i+1} \operatorname{erf}\left(\frac{x-x_{i}}{r_{i}}\right),
$$

where $x$ is the depth, $C_{1}$ and $C_{2}$ the maximum and minimum concentrations of the respective $\mathrm{Ge}$ isotope, and erf the error

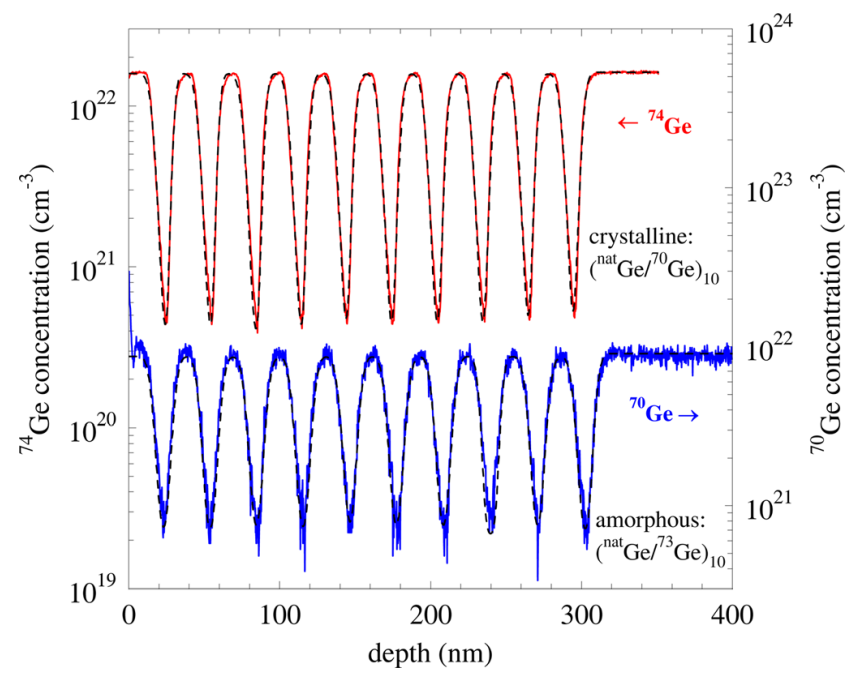

FIG. 1. (Color online) SIMS concentration profiles of the stable isotopes ${ }^{74} \mathrm{Ge}$ (upper solid line) and ${ }^{70} \mathrm{Ge}$ (lower solid line) in crystalline ( ${ }^{\text {nat }} \mathrm{Ge} /$ $\left.{ }^{70} \mathrm{Ge}\right)_{10}$ and amorphous $\left({ }^{\text {nat }} \mathrm{Ge} /{ }^{73} \mathrm{Ge}\right)_{10}$ as-grown multilayers, respectively. Note, the left (right) axis refers to the concentration of ${ }^{74} \mathrm{Ge}\left({ }^{70} \mathrm{Ge}\right)$. The dashed solid lines represent analytical descriptions of the as-grown isotope profiles with a sum of error functions (see Eq. (1)). function defined as $\operatorname{erf}(-\infty)=-1$ and $\operatorname{erf}(\infty)=+1$. $x_{i}$ represents the position of the $i$ th isotope interface and $r_{i}$ is a measure of the steepness in the Ge concentration at the respective interface between natural and isotopic pure $\mathrm{Ge}$ layers. Fitting the concentration profile given by Eq. (1) to the experimental Ge profiles shown in Fig. 1 yields $r$ values in the range of $3.0-4.5 \mathrm{~nm}$ for both the crystalline and amorphous structure.

Room temperature implantation of $310 \mathrm{keV}$ Ga ions with a dose of $1 \times 10^{15} \mathrm{~cm}^{-2}\left(3.3 \times 10^{14} \mathrm{~cm}^{-2}\right)$ leads to an intermixing of the Ge isotopes as demonstrated in Fig. 2(a) (Fig. 2(b)). The self-atom mixing increases with increasing ion implantation dose. The corresponding distribution of $\mathrm{Ga}$ established after the high-dose (low-dose) implantation is illustrated by the two upper solid (two lower short dashed) profiles in Fig. 3. The experimental Ga profiles are accurately reproduced by TRIM simulations ${ }^{12}$ considering the energy and doses set for implantation (see two upper long
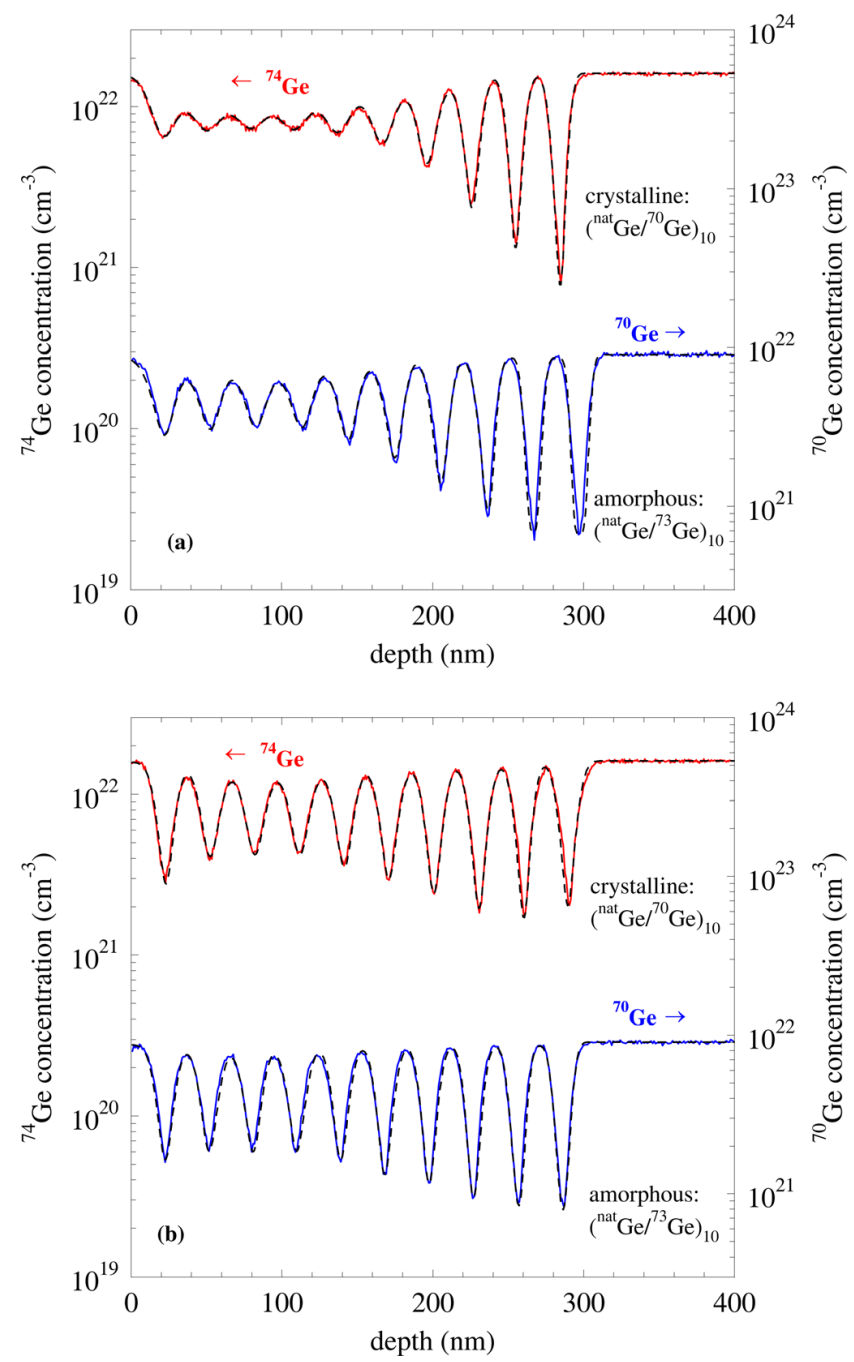

FIG. 2. (Color online) SIMS concentration profiles of ${ }^{74} \mathrm{Ge}$ and ${ }^{70} \mathrm{Ge}$ after implantation of $310 \mathrm{keV} \mathrm{Ga}$ ions with a dose of (a) $1.0 \times 10^{15} \mathrm{~cm}^{-2}$ and (b) $3.3 \times 10^{14} \mathrm{~cm}^{-2}$ in crystalline and amorphous germanium isotope multilayers. The dashed solid lines represent best fits based on the convolution integral (see Eq. (2)). The corresponding distribution of $\mathrm{Ga}$ is illustrated in Fig. 3. 


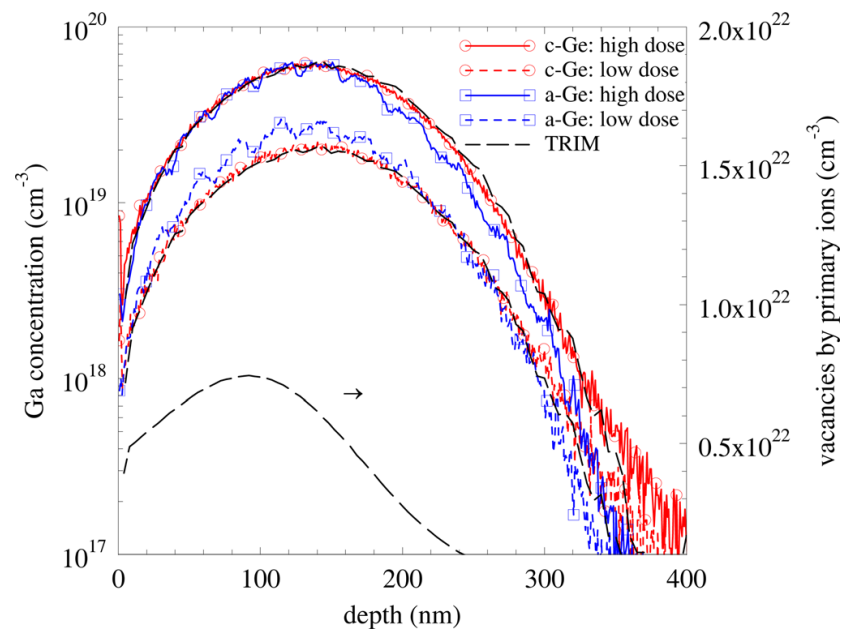

FIG. 3. (Color online) Concentration profiles of Ga measured with SIMS after implantation of $310 \mathrm{keV} \mathrm{Ga}$ ions in crystalline (c-Ge) and amorphous (a-Ge) germanium isotope structures with doses of $1 \times 10^{15} \mathrm{~cm}^{-2}$ (high dose: solid lines) and $3.3 \times 10^{14} \mathrm{~cm}^{-2}$ (low dose: short dashed lines). The TRIM simulation (long dashed lines) of the Ga distribution is in good agreement with the experimental profiles. The lower dashed line shows TRIM results of the concentration of atomic displacements (vacancies) produced by $\mathrm{Ga}$ ions for high-dose implantation.

dashed lines in Fig. 3). Cross-sectional transmission electron microscopy (TEM) analysis of the low- and high-dose Gaimplanted crystalline Ge multilayers were performed to determine the thickness of the amorphous layer formed by Ga implantation.

In addition, crystalline Ge isotope multilayers were implanted at room temperature with $150 \mathrm{keV} \mathrm{Si}$ at a dose of $3 \times 10^{15} \mathrm{~cm}^{-2}$ and $1 \times 10^{15} \mathrm{~cm}^{-2}$. Concentration profiles of ${ }^{74} \mathrm{Ge}$ and ${ }^{70} \mathrm{Ge}$ measured with SIMS after implantation are illustrated in Fig. 4. Again the profiles reveal an increasing intermixing with increasing ion dose.

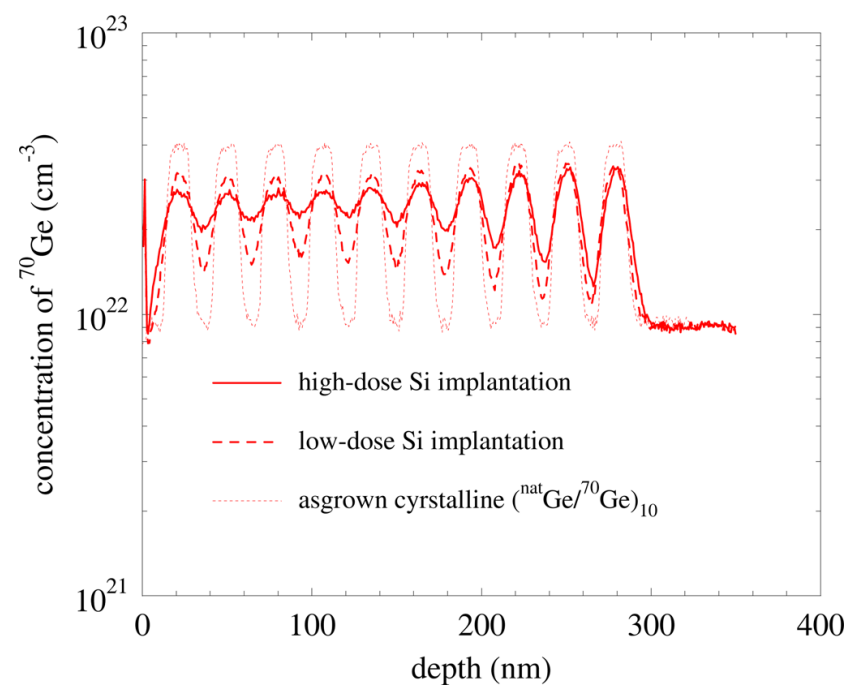

FIG. 4. (Color online) Concentration profiles of ${ }^{70} \mathrm{Ge}$ measured with SIMS after implantation of crystalline germanium isotope multilayers with $150 \mathrm{keV} \mathrm{Si}$ ions and doses of $3 \times 10^{15} \mathrm{~cm}^{-2}$ (solid lines) and $1 \times 10^{15} \mathrm{~cm}^{-2}$ (dashed lines). The stronger intermixing of the isotope structure with increasing dose is evident. The thin short dashed line represents the ${ }^{70} \mathrm{Ge}$ distribution of the as-grown Ge multilayer.
It is noted that all implantations were performed at $7^{\circ}$ tilt angle with respect to the normal of the (100) surface. This avoids channeling effects in crystalline multilayers.

\section{RESULTS AND DISCUSSION}

The Ga profiles illustrated in Fig. 3 reveal their maximum concentration at a depth of about $140 \mathrm{~nm}$. In contrast, the maximum broadening of the Ge isotope multilayer is at about $90 \mathrm{~nm}$ as shown by Fig. 2. This reflects that most crystal damage is formed by cascades initiated by Ga atoms before they come to rest. This is in accord with the prediction of simulations performed by the BCA code TRIM, ${ }^{12}$ which assumes an amorphous target at zero temperature. In the case of the crystalline multilayers the agreement between SIMS data and simulations shows that channeling effects are negligible. According to TRIM the maximum number of atomic displacements by the $\mathrm{Ga}$ ions occurs at a depth of about $90 \mathrm{~nm}$ (see lower long dashed line in Fig. 3). Hence the maximum broadening of the $\mathrm{Ge}$ isotope multilayer correlates with the maximum number of ballistically produced atomic defects.

Figure 2 illustrates that the self-atom mixing induced by $310 \mathrm{keV} \mathrm{Ga}$ ions is stronger in crystalline compared to amorphous Ge. In contrast, the corresponding Ga profiles are very similar (see upper lines in Fig. 3). This result is highly unexpected because ion-beam mixing in amorphous materials is expected to be as large or larger than in its crystalline counterparts. $^{5,11,13}$ In particular, MD simulations of Nordlund et $a l .{ }^{11}$ predict that the magnitude of mixing in amorphous $\mathrm{Ge}$ is about a factor of 2 larger than in crystalline Ge. According to the computer simulations this difference derives from different degree of atomic motion during the relaxation process that follows the collisional phase of the cascade. The mixing process deduced from MD simulations is not supported by the experimental results shown in Fig. 2 that clearly demonstrate a lower ion beam mixing in amorphous Ge than in its crystalline counterpart.

The differences in ion-beam mixing of crystalline and amorphous Ge can be evaluated more quantitatively by modeling the experimental profiles with a convolution integral ${ }^{9}$

$$
C_{\mathrm{Ge}}^{\mathrm{after}}(x)=\int C_{\mathrm{Ge}}^{\mathrm{before}}\left(x^{\prime}\right) \cdot g\left(x-x^{\prime}\right) \cdot d x^{\prime}
$$

where $C_{\mathrm{Ge}}^{\mathrm{before}}$ and $C_{\mathrm{Ge}}^{\mathrm{after}}$ represent the Ge distribution before and after Ga implantation, respectively. $g$ denotes a Gaussian function of the form

$$
g(x)=\frac{1}{\sqrt{2 \pi \sigma}} \exp \left(-\frac{x^{2}}{2 \sigma^{2}}\right) .
$$

The parameter $\sigma$ determines the width of the Gaussian function and is assumed to be depth dependent

$$
\sigma(x)=k \cdot \exp \left(-\frac{(x-l)^{2}}{2 m^{2}}\right)
$$

With Eqs. (2)-(4) the depth dependent ion-beam mixing of the Ge multilayer is described by means of a depth 
dependent $\sigma$. The parameters $k, l$, and $m$ are the amplitude, position, and width of the $\sigma$ function. $\sigma(x)$ accounts for a depth-dependent distribution of the Ge displacement induced by Ga implantation. The parameters $k, l$, and $m$ are optimized to accurately describe the self-atom profiles shown in Fig. 2. The dashed lines are best fits based on Eq. (2) with $\sigma(x)$ profiles illustrated in Fig. 5. The corresponding $k, l$, and $m$ parameters are summarized in Table I. Figure 5 clearly reveals a stronger displacement in crystalline than in amorphous Ge for both the high- and low-dose Ga implantation. TEM analyses of the Ga-implanted crystalline Ge samples reveal amorphous top layers with thicknesses of $(325 \pm 2) \mathrm{nm}$ and $(323 \pm 3) \mathrm{nm}$ for the high- and low-dose implantation, respectively. Substituting these values as depth $x$ in Eq. (4) and considering the respective data for $k, l$, and $m$ (see Table I), a displacement of $(0.73 \pm 0.12) \mathrm{nm}$ and $(0.82 \pm 0.09) \mathrm{nm}$ is obtained. This critical length for $\mathrm{Ge}$ displacement is required to turn the crystalline structure to an amorphous state. This length is in accord with the value of $0.75 \mathrm{~nm}$ reported by Kawamura et al. ${ }^{10}$ from experiments on arsenic implantation induced self-atom mixing in crystalline Ge. The critical displacement length is independent of the incident ion and hence specific for the underlying material system (in Si the value is $0.5 \mathrm{~nm}$, Ref. 9).

Figure 5 shows that the maximum displacement in the amorphous structure occurs at a depth of about $90 \mathrm{~nm}$. This depth coincides with TRIM calculations of the maximum number of vacancies produced by the primary ions (see lower dashed line in Fig. 3). The differences in the position of the maximum displacement observed in Fig. 5 for the crystalline structure after high and low dose Ga implantation is likely related to its transformation to an amorphous phase. Although both samples are crystalline at the beginning they turn amorphous during implantation. Thereby the low dose sample lasts relatively longer with respect to the total time of implantation in a crystalline state than the high dose sample,

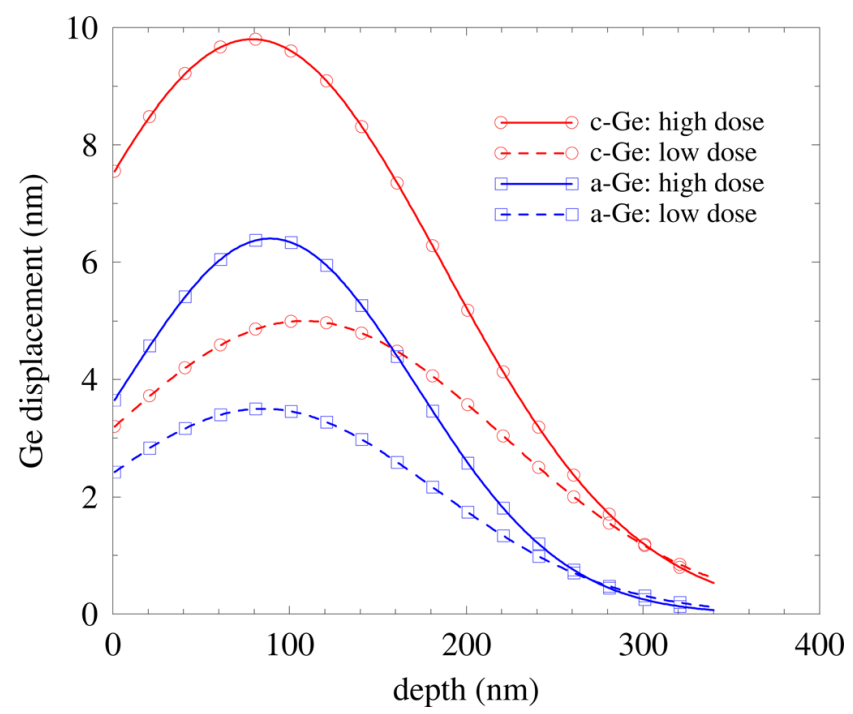

FIG. 5. (Color online) Ge displacement induced by high-dose (solid lines) and low-dose (dashed lines) Ga implantation in crystalline (c-Ge) and amorphous (a-Ge) germanium isotope multilayers. The Ge displacement is higher in crystalline than in amorphous Ge for both doses.
TABLE I. Parameters $k, l$, and $m$ of the depth dependent displacement function $\sigma(x)$ (see Eq. (4)) deduced from the analysis of low (l) and high (h) dose Ga-implanted crystalline (c) and amorphous (a) Ge isotope samples (S).

\begin{tabular}{lllr}
\hline \hline Sample & $k(\mathrm{~nm})$ & $l(\mathrm{~nm})$ & $m(\mathrm{~nm})$ \\
\hline $\mathrm{S}_{c, h}$ & $9.8 \pm 0.2$ & $79 \pm 3$ & $108 \pm 3$ \\
$\mathrm{~S}_{c, l}$ & $5.0 \pm 0.2$ & $97 \pm 3$ & $120 \pm 3$ \\
$\mathrm{~S}_{a, h}$ & $6.4 \pm 0.2$ & $89 \pm 3$ & $83 \pm 3$ \\
$\mathrm{~S}_{a, l}$ & $3.5 \pm 0.2$ & $85 \pm 3$ & $98 \pm 3$ \\
\hline \hline
\end{tabular}

i.e., the high-dose Ga implantation mostly took place in an amorphous phase.

The TRIM simulation of the Ga distribution after $310 \mathrm{keV}$ Ga ion implantation is in excellent agreement with the experimental Ga profile measured with SIMS (see Fig. 3). No significant difference between the implanted Ga profile in crystalline and amorphous Ge is observed. Obviously, the BCA approach, i.e., the assumption that the whole ion implantation process can be described by a sequence of binary collisions between moving projectiles and target atoms at rest, provides good estimates for the primary ion distribution. In contrast, the self-atom mixing induced by $\mathrm{Ga}$ implantation varies significantly for crystalline and amorphous Ge (see Fig. 2). In order to check the prediction of $\mathrm{BCA}$ with respect to ion-beam induced self-atom mixing we performed atomistic simulations by means of the TRIDYN code. ${ }^{14}$ Similarly to TRIM, the BCA program TRIDYN considers an amorphous target at zero temperature, but it treats the dynamic change of the target composition during the implantation process. Figure 6 displays TRIDYN simulations of $\mathrm{Ga}$ and ${ }^{70} \mathrm{Ge}$ atom profiles in comparison to the experimental SIMS profiles obtained after (a) high- and (b) low-dose Ga implantation in crystalline Ge multilayers. Corresponding TRIDYN simulations in comparison to experimental results on $\mathrm{Ga}$ and $\mathrm{Ge}$ distribution in amorphous $\mathrm{Ge}$ are displayed in Fig. 7. As in the case of TRIM the Ga implantation profile is accurately reproduced by TRIDYN (see lower solid and dashed lines in (a) and (b) of Figs. 6 and 7). Taking into account the generally accepted displacement energy $E_{d}$ of $15 \mathrm{eV}$ for $\mathrm{Ge}$, the predicted ion-beam mixing of ${ }^{70} \mathrm{Ge}$ atoms (upper thin dashed line in Fig. 6(a) and (b)) strongly differs from the experimental profile (upper thick solid lines in Figs. 6(a) and (b)). With $E_{d}=15 \mathrm{eV}$ the TRIDYN simulation rather predicts a mixing that hardly deviates from the as-grown ${ }^{70} \mathrm{Ge}$ profile (upper thin solid line in Figs. 6(a) and 6(b)). In order to reproduce the experimental self-atom mixing established by Ga implantation into a crystalline (amorphous) isotope multilayer, a displacement energy of $0.1 \mathrm{eV}(0.4 \mathrm{eV})$ must be assumed (upper thick dashed line in (a) and (b) of Fig. 6 (Fig. 7)). The lower ionbeam mixing of the amorphous compared to the crystalline multilayer suggests a factor of 4 higher displacement energy. However, $E_{d}$ values of $0.1 \mathrm{eV}$ and $0.4 \mathrm{eV}$ are certainly physically not justified. Similar TRIDYN simulations of the selfatom mixing in crystalline multilayers induced by $150 \mathrm{keV}$ $\mathrm{Si}$ implantation with doses of $3 \times 10^{15} \mathrm{~cm}^{-2}$ and $1 \times 10^{15}$ $\mathrm{cm}^{-2}$ reproduce experimental Ge profiles when a displacement energy of about $0.1 \mathrm{eV}$ is assumed as illustrated by 

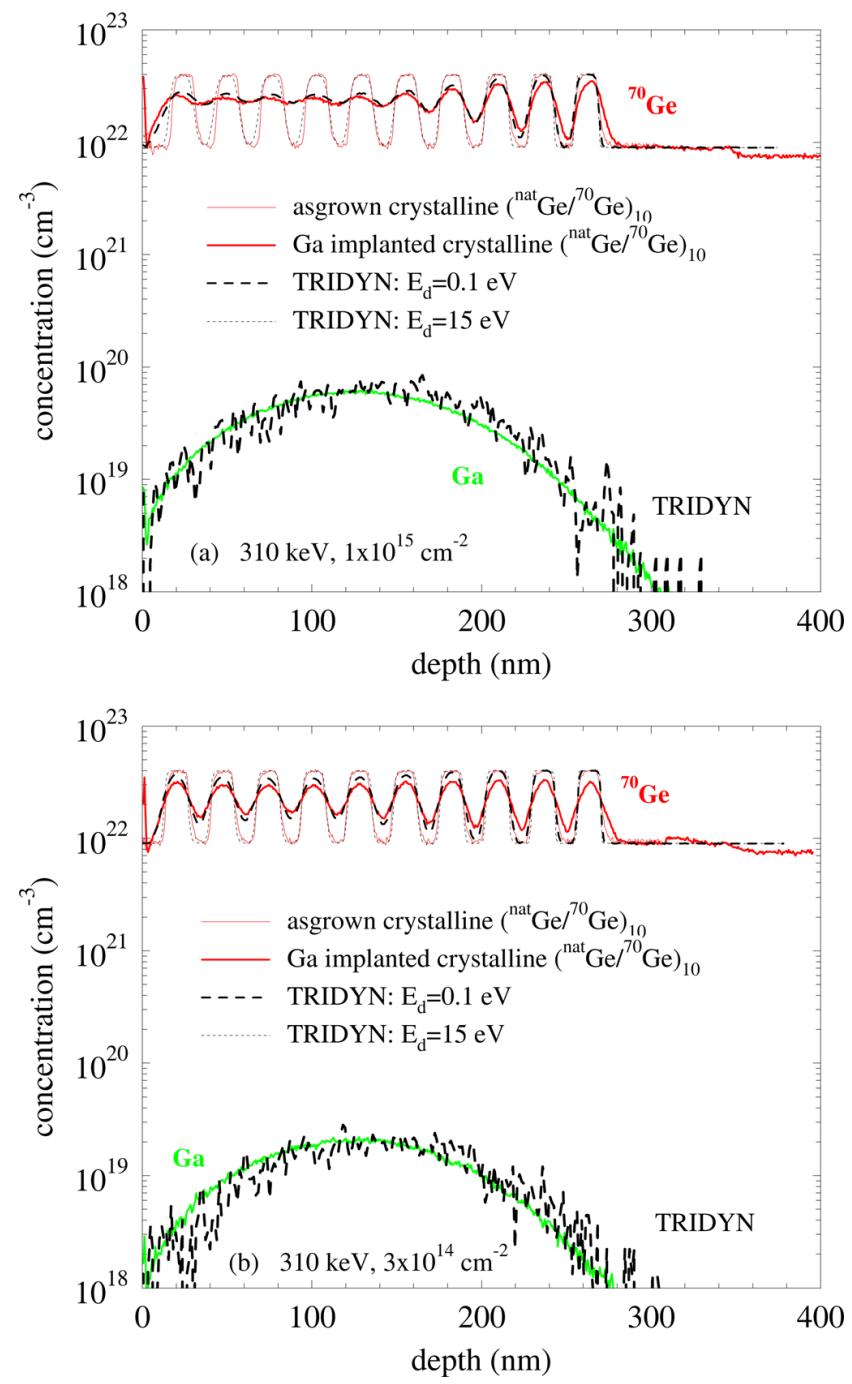

FIG. 6. (Color online) TRIDYN simulations (dashed lines) of the experimental Ga (lower solid lines) and ${ }^{70} \mathrm{Ge}$ atom distribution (upper thick solid lines) in crystalline $\left({ }^{\text {nat }} \mathrm{Ge} /{ }^{70} \mathrm{Ge}\right)_{10}$ multilayers after implantation with $310 \mathrm{keV} \mathrm{Ga}$ ions and a dose of (a) $1 \times 10^{15} \mathrm{~cm}^{-2}$ and (b) $3 \times 10^{14} \mathrm{~cm}^{-2}$. The experimentally observed self-atom mixing is reproduced by TRIDYN when a displacement energy $E_{d}=0.1 \mathrm{eV}$ is assumed (thick dashed lines in (a) and (b)). With the accepted value of $E_{d}=15 \mathrm{eV}$ an intermixing is predicted (upper thin dashed lines in (a) and (b)) that hardly deviates from the asgrown ${ }^{70} \mathrm{Ge}$ profile (upper thin solid lines in (a) and (b)).

Fig. 8. Accordingly, the simulation of self-atom mixing by means of the BCA approach consistently suggests very low energies for the displacement of atoms. There may be different reasons for the unusual low displacement energies. If one assumes that the BCA approach is still applicable and ballistic mixing is still the prevailing phenomenon, the repulsive pair potential used in the simulations might be not correct in the energy range of some tens to some hundreds of $\mathrm{eV}$. These are characteristic energies of the majority of recoil atoms that cause mixing. Indeed the repulsive pair potential was developed to describe atomic collisions at higher energies. Also, experimental data on atomic collisions in the sub$\mathrm{keV}$ region are scarce. However, another reason for the discrepancy should be more convincing. Ballistic mixing alone cannot be responsible and thermal spike mixing should contribute significantly to the observed mixing effects. The im-
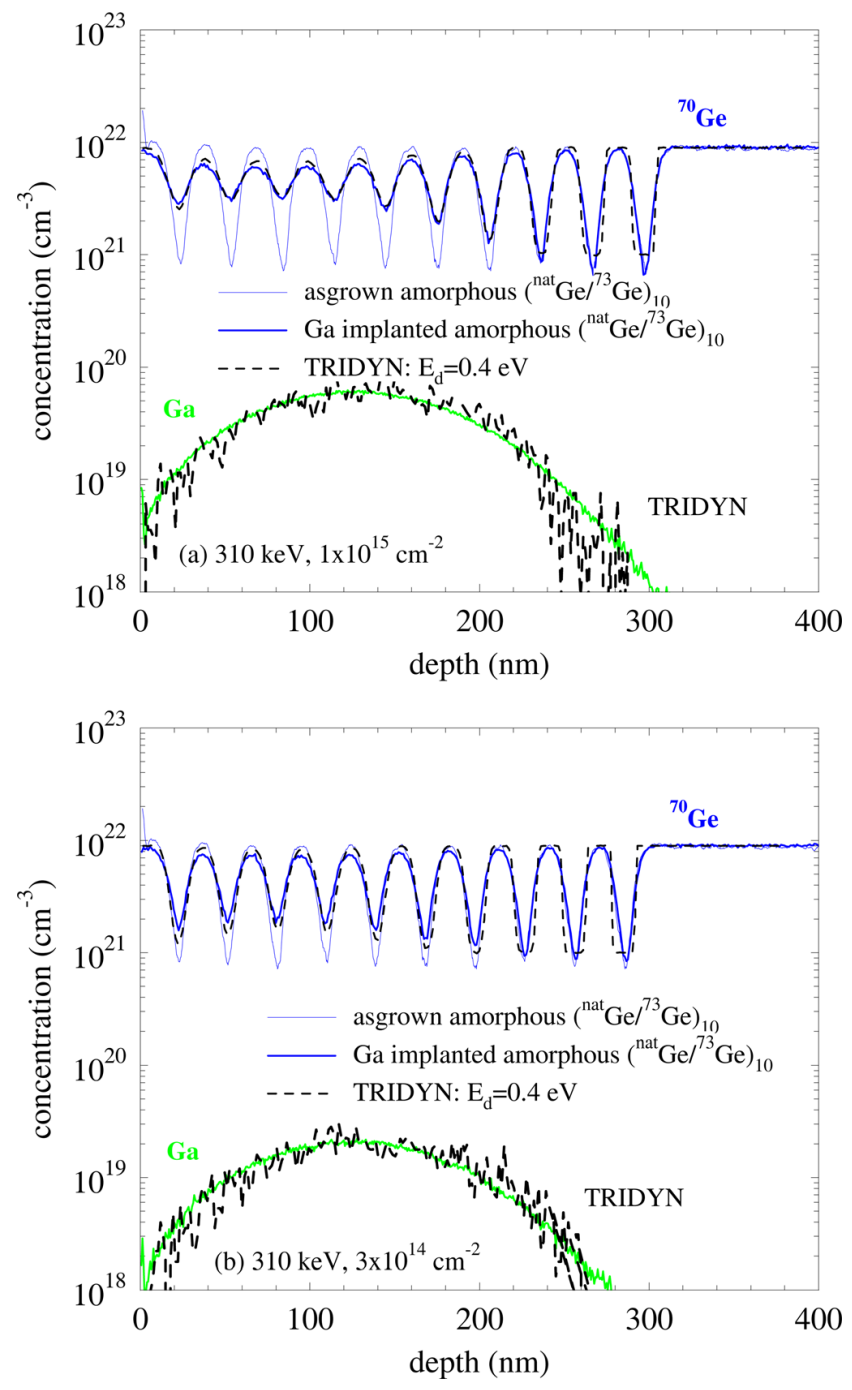

FIG. 7. (Color online) TRIDYN simulations (dashed lines) of the experimental $\mathrm{Ga}$ (lower solid lines) and ${ }^{70} \mathrm{Ge}$ atom distribution (upper thick solid lines) in amorphous $\left({ }^{\text {nat }} \mathrm{Ge} /{ }^{73} \mathrm{Ge}\right)_{10}$ multilayers after implantation with $310 \mathrm{keV} \mathrm{Ga}$ ions and a dose of (a) $1 \times 10^{15} \mathrm{~cm}^{-2}$ and (b) $3 \times 10^{14} \mathrm{~cm}^{-2}$. The experimentally observed self-atom mixing is reproduced by TRIDYN when a displacement energy $E_{d}=0.4 \mathrm{eV}$ is assumed (thick dashed lines in (a) and (b)). With the accepted value of $E_{d}=15 \mathrm{eV}$ an intermixing is predicted (not shown) that hardly deviates from the as-grown ${ }^{70} \mathrm{Ge}$ profile (upper thin solid lines in (a) and (b)).

portance of the thermal spike model for the understanding of damage formation, amorphization and atomic mixing has been already outlined in the literature (see, e.g., Ref. 5). Obviously, the formation of thermal spikes that even lead to amorphous pockets by melting and quenching of small regions should be taken into account in ion-beam mixing of isotopically enriched Ge multilayers. The ultrafast formation and quenching of a liquid-like region should cause significantly more atomic transport than scattered ballistic events. Effects such as collective atomic interactions and melting in regions with a high level of deposited energy density cannot be described theoretically within the framework of the BCA approach.

Assuming that the magnitude of ion-beam mixing in both the crystalline and amorphous Ge isotope multilayers is mainly a consequence of thermal spike effects, the disparity in ion-beam mixing of the crystalline and amorphous 


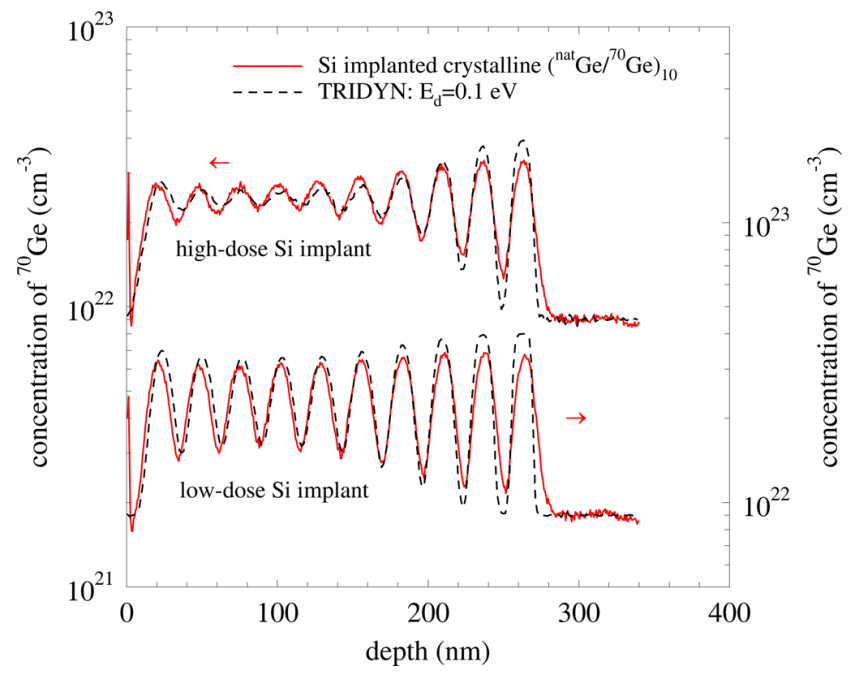

FIG. 8. (Color online) TRIDYN simulations (dashed lines) of the experimental ${ }^{70} \mathrm{Ge}$ distribution (thick solid lines) measured with SIMS after implantation of $150 \mathrm{keV} \mathrm{Si}$ ions in crystalline $\left({ }^{\text {nat }} \mathrm{Ge} /{ }^{70} \mathrm{Ge}\right)_{10}$ multilayers at a dose of $3 \times 10^{15} \mathrm{~cm}^{-2}$ (upper profiles) and $1 \times 10^{15} \mathrm{~cm}^{-2}$ (lower profiles). The experimentally observed self-atom mixing is reproduced by TRIDYN when a low displacement energy $E_{d}=0.1 \mathrm{eV}$ is assumed.

structures indicate fundamental differences in the mechanisms of mixing. Strong differences must exist in the early stages of self-atom mixing when the crystalline Ge has not turned amorphous. Presumably, the mixing in the crystalline multilayers at the early stage is mainly controlled by radiation enhanced diffusion (RED) of self-atoms in still crystalline but highly damaged regions of the collision cascades. Certainly, local melting of the crystalline structure may also occur during thermal spike formation but obviously the contribution of self-atom mixing in local amorphous pockets does not exceed that of RED. The RED is limited by the migration enthalpy of the native point defects formed by the incident ion. In this context we refer to recent experiments on RED in Ge that demonstrate strong athermal RED effects, i.e., increasing RED with decreasing temperature. This unusual behavior is related to the presence of highly mobile Ge interstitials, ${ }^{15,16}$ whose concentration increases with decreasing temperature due to the decreasing number of vacancies available for annihilation. ${ }^{17}$ Finally, with continuing ion-beam treatment, the crystalline matrix becomes amorphous and the mixing mechanism changes. Self-atom mixing in amorphous Ge that is likely associated with thermal spike formation may be mainly mediated by cooperative diffusion events involving several matrix atoms. Differences in the density of the amorphous and crystalline Ge matrix $^{18,19}$ can favor more localized collision cascades in the amorphous compared to the crystalline matrix and thus lower self-atom mixing. Furthermore the ion-beam induced amorphization process itself, which does only occur in the crystalline multilayers, may contribute to the observed difference. Still the higher ion-beam induced mixing of crystalline compared to amorphous Ge remains surprising because crystalline Ge implanted with $\mathrm{Ga}$ at high-dose should be most of the implantation time in an amorphous state. Obviously, the intermixing in the crystalline state is so enormous that a memory effect of the initial state is apparent even after high-dose implants.
Although the present results cannot unravel conclusively the underlying mechanisms of self-atom mixing in crystalline and amorphous Ge, they stimulate further experimental and theoretical studies. In particular, experiments on the temperature dependence of ion-beam induced self-atom mixing in crystalline and amorphous Ge and on the early stages of intermixing could clarify the contribution of point defects, cooperative diffusion events, and ballistic effects in the overall mixing efficiency. Moreover, MD calculations of the early stage of ion-beam mixing in crystalline and amorphous Ge could help to verify different mechanisms of self-atom mixing. Present MD simulations rather predict larger ionbeam mixing in amorphous semiconductors compared to its crystalline counterpart. ${ }^{11}$

\section{CONCLUSION}

Gallium implantation induced mixing of crystalline and amorphous Ge isotope multilayer structures was investigated by means of secondary ion mass spectrometry. A convolution integral method was applied for the analysis of the $\mathrm{Ga}$ ion-beam induced self-atom mixing. An average displacement length of $0.78 \mathrm{~nm}$ for ion-beam amorphization of $\mathrm{Ge}$ was determined that confirms recent results of Kawamura et $a l .{ }^{10}$ Atomistic simulations based on the binary collision approximation reproduce the degree of ion beam mixing induced by $\mathrm{Ga}$ as well as $\mathrm{Si}$ implantation in Ge only if unphysically low Ge displacement energies are assumed. The consistently observed strong ion-beam mixing suggests a significant contribution of thermal spikes in the overall mixing of both crystalline and amorphous Ge multilayers. The observed larger ion-beam mixing efficiency in crystalline compared to amorphous $\mathrm{Ge}$ indicates fundamental differences in the mechanisms of self-atom mixing that disproves recent results of MD simulations on the mechanisms of ion-beam mixing in crystalline and amorphous semiconductors. Presumably, the mixing in crystalline $\mathrm{Ge}$ is mainly controlled by radiation enhanced diffusion until the structure becomes amorphous. RED in Ge is dominated by self-interstitials that result from Frenkel pairs formed by irradiation. ${ }^{15,16,20}$ When the solid becomes amorphous, the mixing mechanisms changes. In the amorphous state self-atom mixing is rather mediated by cooperative jump processes than by diffusion events associated with native point defects. In order to understand the remarkable difference in ion-beam mixing of crystalline and amorphous Ge, future investigations will consider the temperature dependence and the early stage of ion-beam induced self-atom mixing.

\section{ACKNOWLEDGMENTS}

The authors thank Tascon GmbH Münster for the SIMS measurements.

${ }^{1}$ R. Collins, Radiat. Eff. Defects Solids 98, 167 (1986).

${ }^{2}$ Y. -T. Cheng, Mater. Sci. Rep. 5, 45 (1990).

${ }^{3}$ M. Nastasi and J. W. Mayer, Mater. Sci. Eng. R. 12, 1 (1994).

${ }^{4}$ W. Bolse, Mater. Sci. Eng., A 253, 194 (1998).

${ }^{5}$ R. S. Averback and T. Diaz de la Rubia, Solid State Phys. 51, 281 (1998).

${ }^{6}$ W. Vandervorst, T. Janssens, B. Brijs, R. Delhougne, R. Loo, M. Caymax, B. J. Pawlak, and M. Posselt, Appl. Phys. Lett. 86, 081915 (2005). 
${ }^{7}$ R. S. Averback, D. Peak, and L. J. Thompson, Appl. Phys. A 39, 59 (1986). ${ }^{8}$ B. V. King, C. Jeynes, R. P. Webb, and J. A. Kilner, Nucl. Instrum. Methods Phys. Res. B 80/81, 163 (1993).

${ }^{9}$ Y. Shimizu, M. Uematsu, K. M. Itoh, A. Takano, K. Sawano, and Y. Shiraki, Appl. Phys. Express 1, 021401 (2008).

${ }^{10}$ Y. Kawamura, Y. Shimizu, H. Oshikawa, M. Uematsu, E. E. Haller, and K. M. Itoh, Phys. B 404, 4546 (2009).

${ }^{11}$ K. Nordlund, M. Ghaly, and R. S. Averback, J. Appl. Phys. 83, 1238 (1998).

${ }^{12}$ J. P. Biersack and L. G. Haggmark, Nucl. Instrum. Methods 174, 257 (1980).

${ }^{13}$ D. V. Forbes, J. J. Coleman, J. L. Klatt, and R. S. Averback, J. Appl. Phys. 77, 3543 (1995)

${ }^{14}$ W. Möller and W. Eckstein, Nucl. Instrum. Methods Phys. Res. B 2, 814 (1984).

${ }^{15}$ H. Bracht, S. Schneider, J. N. Klug, C. Y. Liao, J. Lundsgaard Hansen, E. E. Haller, A. Nylandsted Larsen, D. Bougeard, M. Posselt, and C. Wündisch, Phys. Rev. Lett. 103, 255501 (2009).
${ }^{16}$ H. Bracht, S. Schneider, and R. Kube, Microelectron. Eng. 88, 452 (2011). ${ }^{17}$ Experiments on radiation enhanced self- and dopant diffusion in Ge (Refs. 15 and 16) reveal that the Ge surface is an insufficient sink for Ge interstitials, whereas vacancies are readily annihilated. As a consequence, an enormous supersaturation of self-interstitials is established during irradiation, whereas the vacancy concentration is close to thermal equilibrium. With decreasing temperature the vacancy concentration in thermal equilibrium decreases and with it the annihilation sites for Ge interstitials. As a consequence the supersaturation of Ge interstitials increases with decreasing temperature. This leads to an athermal RED, i.e., an increased RED with decreasing temperature.

${ }^{18}$ J. R. Blanco, P. J. McMarr, J. E. Yehoda, K. Vedam, and R. Messier, J. Vac. Sci. Technol. A 4, 577 (1986).

${ }^{19}$ M. Posselt and A. Gabriel, Phys. Rev. B 80, 045202 (2009).

${ }^{20}$ E. Bruno, S. Mirabella, G. Scapellato, G. Impellizzeri, A. Terrasi, F. Priolo, E. Napolitani, D. De Salvador, M. Mastromatteo, and A. Carnera, Phys. Rev. B 80, 033204 (2009). 